\title{
Simultaneous Temperature and Strain Sensing for Cryogenic Applications Using Dual-Wavelength Fiber Bragg Gratings
}

\author{
Meng-Chou Wu*, William H. Prosser \\ NASA, Langley Research Center, MS 231, Hampton, VA, USA 23681-2199
}

\begin{abstract}
A new technique has been developed for sensing both temperature and strain simultaneously by using dual-wavelength fiber-optic Bragg gratings. Two Bragg gratings with different wavelengths were inscribed at the same location in an optical fiber to form a sensor. By measuring the wavelength shifts that resulted from the fiber being subjected to different temperatures and strains, the wavelength-dependent thermo-optic coefficients and photoelastic coefficients of the fiber were determined. This enables the simultaneous measurement of temperature and strain. In this study, measurements were made over the temperature range from room temperature down to about $10 \mathrm{~K}$, addressing much of the low temperature range of cryogenic tanks. A structure transition of the optical fiber during the temperature change was found. This transition caused splitting of the waveforms characterizing the Bragg gratings, and the determination of wavelength shifts was consequently complicated. The effectiveness and sensitivities of these measurements in different temperature ranges are also discussed.
\end{abstract}

Keywords: fiber Bragg gratings, fiber optic sensors, cryogenic applications

\section{INTRODUCTION}

Fiber Bragg gratings (FBGs) have been widely used as strain sensors in aerospace applications, as well as in other fields. ${ }^{1-3}$ Compared with conventional strain gauges, FBG strain sensors have similar sensitivities but are much lighter in weight and require simpler wiring. These advantages are especially important for aerospace applications. However, FBGs are also sensitive to temperature changes. Thermally induced wavelength shifts of a FBG are indistinguishable from those induced by strain within a simple strain measurement. This technical issue can be relatively serious when the measurements are conducted in an environment with large temperature gradients or fluctuations, for example, on the surface of cryogenic fuel tanks employed in aerospace vehicles. In these cases temperature compensation is necessary. There have been several techniques developed for intrinsic temperature compensation. ${ }^{4-8} \mathrm{Xu}$ et al. ${ }^{4}$ have demonstrated a method using dual-wavelength gratings to simultaneously measure temperature and strain. In this method a sensing element contains two superimposed FBGs, written at $850 \mathrm{~nm}$ and $1300 \mathrm{~nm}$. The thermo-optic coefficients and photoelastic coefficients are wavelength-dependent and the large center wavelength separation therefore allows differentiating between the thermal and strain effects. Nevertheless this technique requires two broadband light sources and two detecting systems. This requirement might be impractical for some aerospace applications.

Further, most techniques for temperature compensation address temperature ranges only around and above room temperature. Few investigate the lower temperature range, for example, of cryogenic tanks. To the best of our knowledge, a complete picture for the thermal responses of FBGs is still not available. In this study we investigate the feasibility of cryogenic applications using dual-wavelength FBGs for strain/temperature discrimination. The gratings were written around $1550 \mathrm{~nm}$ with a separation of $30 \mathrm{~nm}$, such that only a single scanning laser is required for the experiment. Measurements were made over the temperature range from room temperature down to about $10 \mathrm{~K}$, addressing much of the low temperature range of cryogenic tanks.

*m.c.wu@larc.nasa.gov 


\section{THEORY}

When using FBG sensors in measurements of strain or temperature, it is assumed that the Bragg wavelength shifts $\Delta \lambda_{\varepsilon}$ and $\Delta \lambda_{T}$ are linear in response respectively to a strain change $\Delta \varepsilon$ and a temperature change $\Delta T$ and can therefore be expressed in the form

$$
\begin{aligned}
& \Delta \lambda_{\varepsilon}=K_{\varepsilon} \Delta \varepsilon \\
& \Delta \lambda_{T}=K_{T} \Delta T
\end{aligned}
$$

for small perturbations, where $K_{\varepsilon}$ and $K_{T}$ are the strain and temperature coefficients respectively. $K_{\varepsilon}$ is determined by the Poisson ratio of the fiber, the photoelastic coefficient and the effective refractive index of the fiber core, and $K_{T}$ by the thermal expansion coefficient and the thermo-optic coefficient. In general, a Bragg wavelength shift is a linear combination of strain and temperature, that is,

$$
\Delta \lambda_{B}(\varepsilon, T)=K_{\varepsilon} \Delta \varepsilon+K_{T} \Delta T
$$

It is also assumed that these individual terms are independent. That is, the related strain-temperature cross-term is negligible, a phenomenon which applies well for small perturbations. For two Bragg wavelength shifts to be observed, the resulting two equations can be expressed in a matrix form,

$$
\left[\begin{array}{l}
\Delta \lambda_{B 1} \\
\Delta \lambda_{B 2}
\end{array}\right]=\left[\begin{array}{ll}
K_{1 T} & K_{2 T} \\
K_{1 \varepsilon} & K_{2 \varepsilon}
\end{array}\right]\left[\begin{array}{c}
\Delta \varepsilon \\
\Delta T
\end{array}\right]
$$

The elements of this $2 \times 2 \mathrm{~K}$ matrix can be determined by measuring each Bragg wavelength shift with strain and temperature separately. With the constructed matrix, the changes of temperature and strain can be calculated by experimentally measuring both of the Bragg wavelength shifts. This can be expressed as the inverse of Eq. (3),

$$
\left[\begin{array}{c}
\Delta \varepsilon \\
\Delta T
\end{array}\right]=C\left[\begin{array}{cc}
K_{2 \varepsilon} & -K_{1 \varepsilon} \\
-K_{2 T} & K_{1 T}
\end{array}\right]\left[\begin{array}{c}
\Delta \lambda_{B 1} \\
\Delta \lambda_{B 2}
\end{array}\right]
$$

where $\mathrm{C}=1 /\left(K_{1 T} K_{2 \varepsilon}-K_{2 T} K_{1 \varepsilon}\right)$, The condition that $\mathrm{C}$ cannot be zero requires that the ratio of the strain responses of two gratings be different from the ratio of their temperature responses, i.e., $K_{1 T} / K_{2 T} \neq K_{1 \varepsilon} / K_{2 \varepsilon}$.

\section{EXPERIMENT}

A pulsed Excimer laser of $248 \mathrm{~nm}$ and a Talbot interferometer arrangement were used to write the dual-wavelength FBGs, as shown in Fig. 1. Both standard telecommunication fiber and boron-germanium co-doped photosensitive fiber were used. The wavelengths of gratings were adjusted by changing the relative angle of the two mirrors in the interferometer. For writing a dual-wavelength grating, two gratings were superimposed and their wavelengths were maintained around $1550 \mathrm{~nm}$ with a separation of about $30 \mathrm{~nm}$. Typical exposure was a $20 \mathrm{~Hz}$ pulse rate for a duration of less than 1 minute. This produced gratings with relatively low reflectivities, which might be difficult to be monitored with a conventional optical spectrum analyzer.

The low reflectivity gratings were interrogated by using a frequency domain demodulation system ${ }^{9}$, shown in Fig. 2. In this system, the fiber coupler C1 and a pair of Faraday rotation mirrors (FRM's) form an in-fiber interferometer with an optical path difference of $2 n_{\text {eff }} \mathrm{L}_{0}$, where $n_{\text {eff }}$ is the effective refractive index of the fiber core and $\mathrm{L}_{0}$ the length of the reference cavity. The signals are driven by the tuning of the laser and detected at the photo-detector $\mathrm{D}_{1}$. They are used to trigger the sampling of signal at $\mathrm{D}_{2}$, which is the output of another in-fiber interferometer formed with the fiber coupler $\mathrm{C}_{2}$, a broadband reflector, and a particular fiber Bragg grating at a distance of $\mathrm{L}_{\mathrm{i}}$. A series of low reflectivity Bragg 
gratings can be written at the same wavelength on a single fiber at different locations and the reflected signals are superimposed and detected at $\mathrm{D}_{2}$.

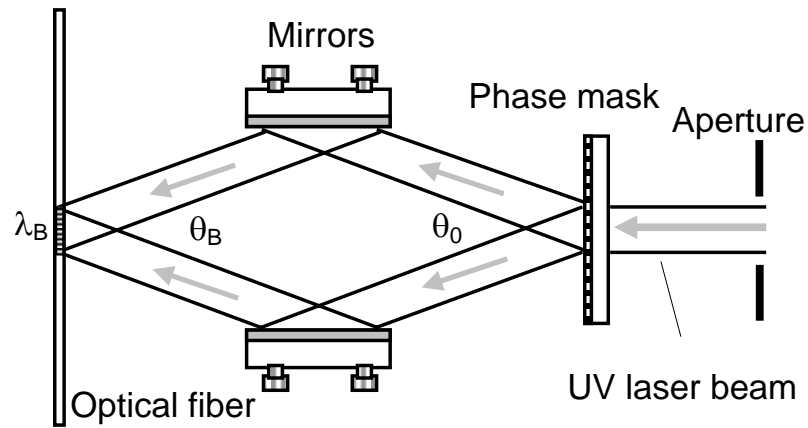

Figure 1: Schematic representing the interferometric technique for generating fiber Bragg gratings. The Bragg wavelength $\lambda_{B}$ is determined by the adjustable $\theta_{B}$.

The detected signals are further processed (fast-Fourier-transformed) to display the spatial spectrum, which shows the distances and physical profiles of the gratings. The spectrum of a particular grating can then be windowed and inversefast-Fourier-transformed to get its wavelength spectrum. Shown in Fig. 3(a) is the spatial spectrum of a typical FBG with a physical length of about $3 \mathrm{~mm}$ and (b) its wavelength spectrum, which can be used for strain and temperature sensing. In Fig. 4(a), the spatial spectrum of a dual-wavelength Bragg grating shows the characteristics of interference by two superimposed gratings. Fig. 4(b) shows the wavelength spectrum of this dual-wavelength Bragg grating, with nominal Bragg wavelengths of $\sim 1536 \mathrm{~nm}$ and $\sim 1564 \mathrm{~nm}$.

A cryostat of compressed helium gas was used for temperature control. After the gratings had been written, the stripped portion of the optical fiber was not recoated and directly attached to the cold plate in the cryogenic chamber.

Temperature was varied from slightly above room temperature down to about $10 \mathrm{~K}$ and controlled within $0.05 \mathrm{~K}$. The center wavelengths were monitored using the processed signals from the frequency domain demodulation system described above, with a resolution of $0.02 \mathrm{~nm}$.

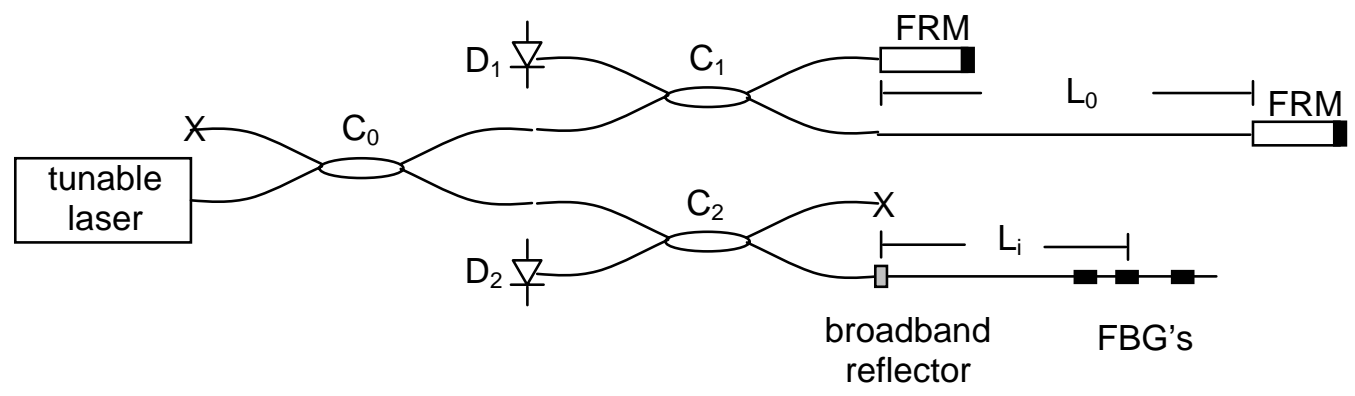

Figure 2: Schematic diagram of a frequency domain demodulation system. Items $\mathrm{C}_{0}, \mathrm{C}_{1}$, and $\mathrm{C}_{2}$ are fiber couplers. " $\mathrm{X}$ " indicates that the unused port is terminated. Items D1 and D2 are detectors and FRM's a pair of Faraday rotation mirrors. 

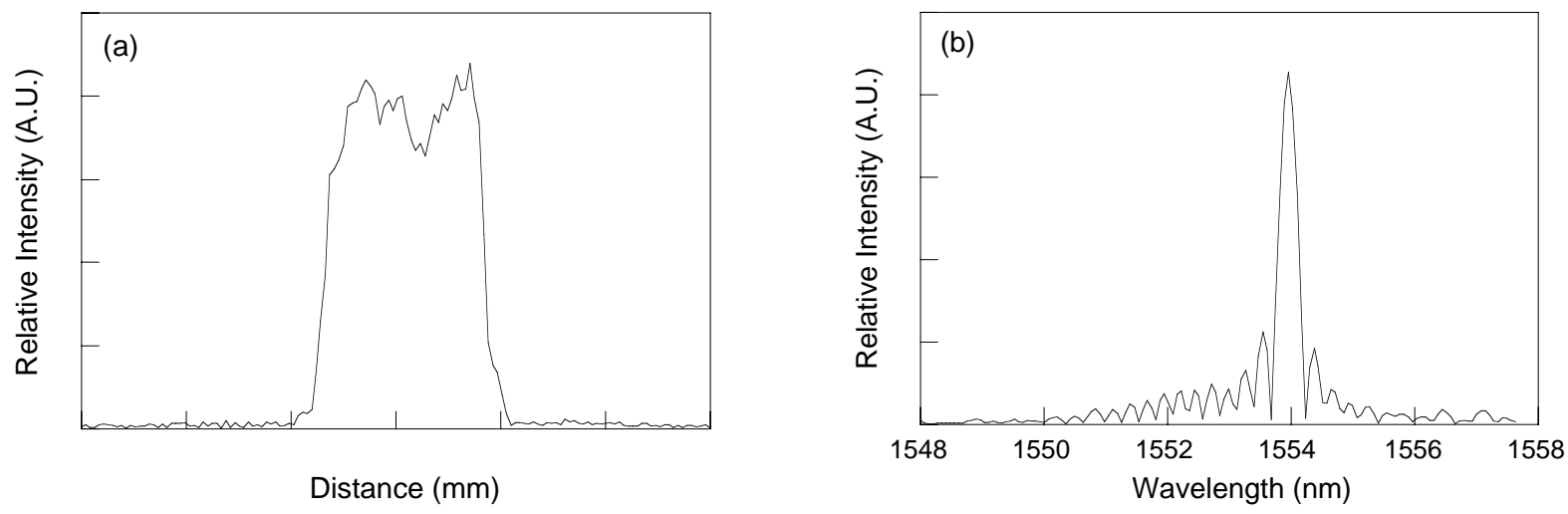

Figure 3: (a) Spatial spectrum and (b) wavelength spectrum of a written Bragg grating with a physical length of about $3 \mathrm{~mm}$ and a center wavelength of $1554 \mathrm{~nm}$.
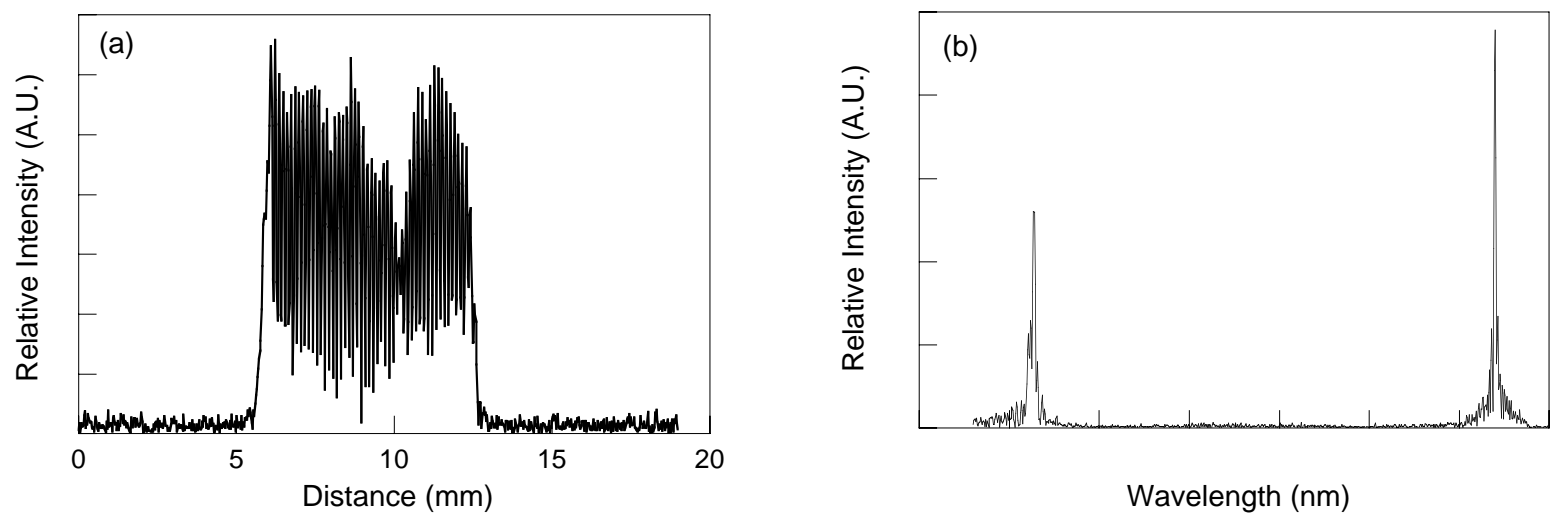

Figure 4: (a) Spatial spectrum and (b) wavelength spectrum of a dual-wavelength Bragg grating with nominal Bragg wavelengths of about $1536 \mathrm{~nm}$ and $1564 \mathrm{~nm}$.

\section{RESULTS AND DISCUSSION}

Fig. 5 and Fig. 6 show the temperature responses of Bragg gratings. It is clear that the thermally-induced wavelength shifts are relatively linear from $310 \mathrm{~K}$ down to $160 \mathrm{~K}$, although there are a few slight changes of gradients within this temperature range. This result of linearity in a finite range is consistent with the assumption that the temperature coefficient is linear for small perturbations.

Fig. 7(a) and (b) show the linear curve fitting of the thermal responses at two Bragg wavelengths for the temperature range from $244 \mathrm{~K}$ to $312 \mathrm{~K}$. The measured temperature coefficients were

$$
\begin{aligned}
& K_{1 T}=8.07 \pm 5.4 \times 10^{-2} \mathrm{pm} /{ }^{\circ} \mathrm{C} \\
& K_{2 T}=8.30 \pm 5.0 \times 10^{-2} \mathrm{pm} /{ }^{\circ} \mathrm{C}
\end{aligned}
$$

where the temperature scale was converted to conventional degree Celsius. It should be noted that these values are of bare fibers without the contribution of coating materials, as mentioned in the previous section. 
From $150 \mathrm{~K}$ to $80 \mathrm{~K}$, the changes are more complicated. The original well-defined center peak of the Bragg wavelength is split. The determination of the wavelength shift is therefore more difficult, if not impractical. This behavior is possibly due to the structure transition of germanium-induced defect levels. Further investigation needs to be done. As the temperature approaches the transition temperature, the nonlinearity of the responses makes the dual-wavelength Bragg gratings impractical for the measurement of temperature.

For the temperature range from $80 \mathrm{~K}$ to $10 \mathrm{~K}$, the Bragg wavelength has practically no thermally-induced changes, due to extremely low thermal expansion coefficient and thermo-optic coefficient. It is obviously impossible to use the dualwavelength Bragg gratings to determine the temperature. However, in this temperature range, the wavelength shifts can be totally attributed to the effect of strain, i.e., strains can be measured without the corrections for temperature.

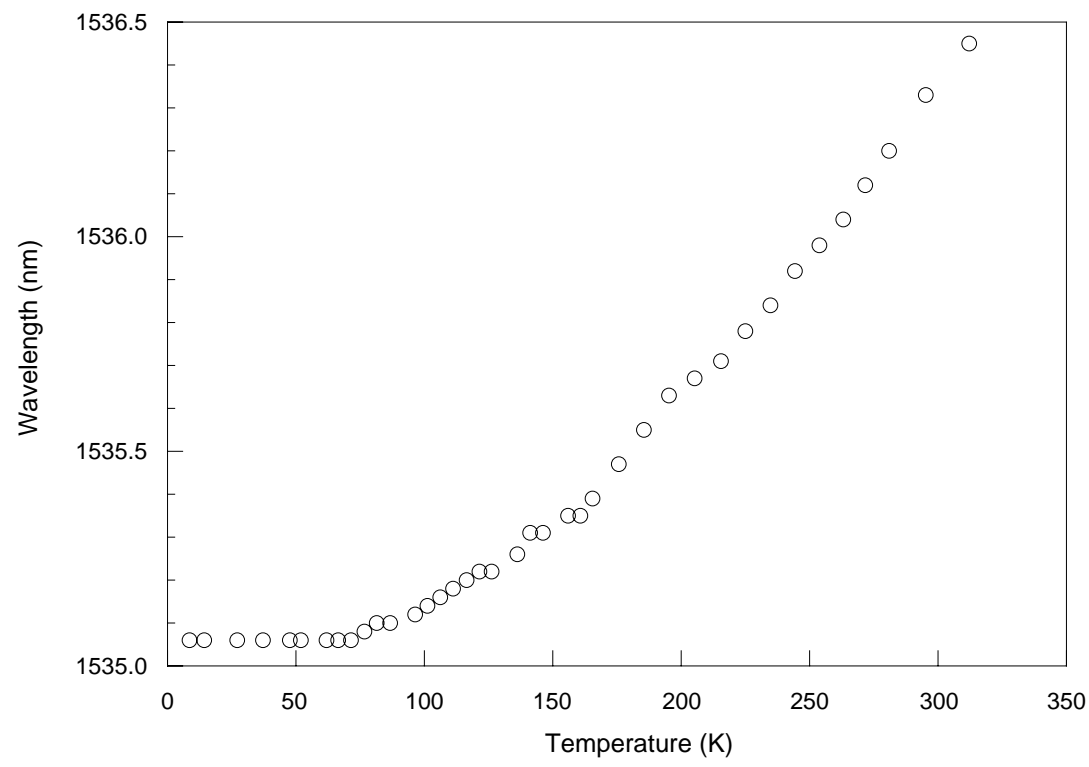

Figure 5: Temperature response of the Bragg grating with a nominal wavelength of $1536 \mathrm{~nm}$.

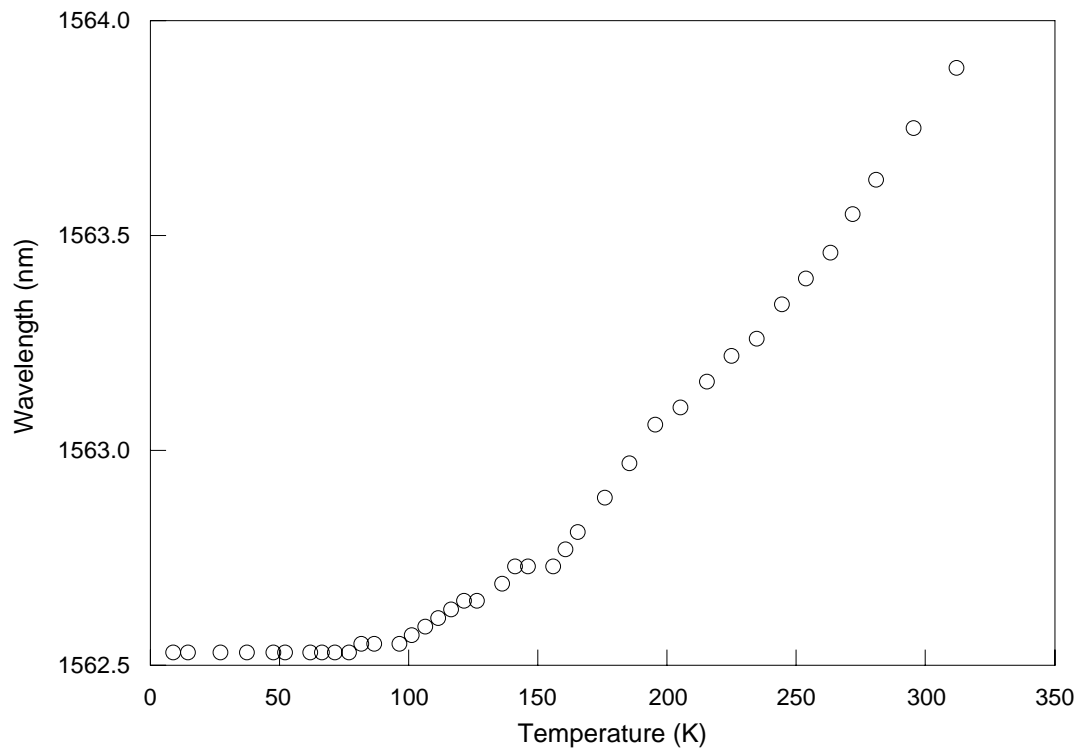

Figure 6: Temperature response of the Bragg grating with a nominal wavelength of $1564 \mathrm{~nm}$. 

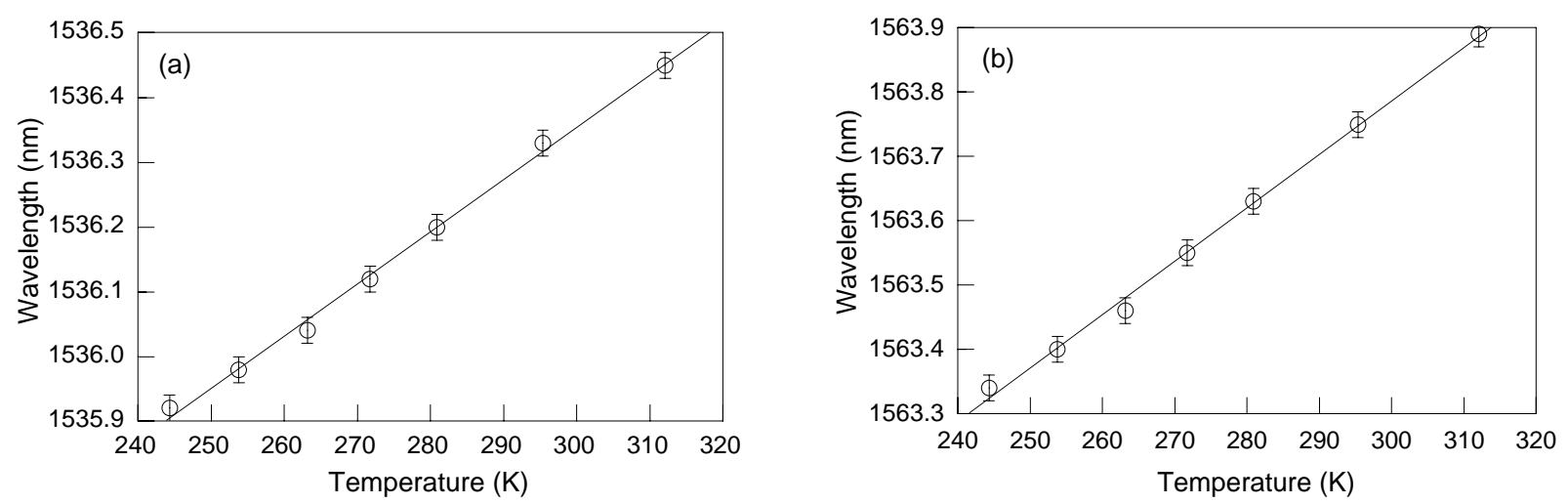

Figure 7: Linear curve fitting of the thermal responses at two Bragg wavelengths for the temperature range from $244 \mathrm{~K}$ to $312 \mathrm{~K}$.

\section{SUMMARY}

A new technique, using low reflectivity FBGs for strain/temperature discrimination, is demonstrated. In this study, measurements were made over the temperature range from room temperature down to about $10 \mathrm{~K}$, addressing much of the low temperature range of cryogenic tanks. Linear thermal responses of these gratings were found in the higher temperature range. A possible structure transition of the optical fiber was found as the temperature decreased. This transition caused splitting of the waveforms characterizing the Bragg gratings, and the determination of wavelength shifts was consequently complicated. For temperatures lower than $90 \mathrm{~K}$, the temperature coefficients of FBGs were found to be practically zero and hence strains can be measured without temperature compensations. Further studies will be conducted for strain measurements.

\section{REFERENCES}

1. C. R. Giles, "Lightwave applications of fiber Bragg gratings," J. Lightwave Technol. 15, 1391-1404 (1997)

2. K. O. Hill, Y. Fujii, D. C. Johnson, and B. S. Kawasaki, "Photosensitivity in optical waveguides: application to reflection filter fabrication, “Appl. Phys. Lett. 32, 647-649 (1978)

3. A. D. Kersey, M. A Davis, H. J. Patrick, M. LeBlanc, K. P. Koo, C. G. Askins, M.A. Putnam, E. J. Friebele, "Fiber grating sensors," J. Lightwave Technol. 15, 1442-1463 (1997)

4. M. G. Xu, J.-L. Archambault, L. Reekie, and J. P. Dakin, "Discrimination between strain and temperature effects using dual-wavelength fiber grating sensors," Electron. Lett. 31, 823-825 (1994).

5. S. W. James, M. L. Dockney, and R. P. Tatum, "Simultaneous independent temperature and strain measurement using in-fiber Bragg grating sensors," Electron. Lett. 32, 1133-1134 (1996).

6. H. J. Patrick, G. M. Williams, A. D. Kersey, and J. R. Pedrazzani, "Hybrid fiber Bragg grating/long period fiber grating sensor for strain/temperature discrimination,” IEEE Photon. Technol. Lett. 8, 1223-1225 (1996).

7. S. E. Kannellopoulos, V. A. Handrek, and A. J. Rogers, "Simultaneous strain and temperature sensing with photogenerated in-fiber gratings," Optic. Lett. 20, 333-335 (1995)

8. G. P. Brady, K. Kalli, D. J. Webb, D.A. Jackson, L. Reekie, an J.-L. Archambault, "Simultaneous measurement of strain and temperature using the first- and second-order diffraction wavelengths of Bragg gratings," IEE Proc.Optoelectron. 144, 1556-161 (1997)

9. M. Froggatt and J. Moore, "Distributed measurement of static strain in an optical fiber with multiple Bragg gratings at nominally equal wavelengths." Appl. Opt. 37, 1741-1746 (1998). 